\title{
Control strategies for line following applied to robots with nonholonomic constraint
}

\author{
Thales Silva* Victor Hugo Pereira Rodrigues* Fernando Lizarralde * \\ * Programa de Engenharia Elétrica, COPPE \\ Universidade Federal do Rio de Janeiro
}

\begin{abstract}
In this paper the line-following problem for a nonholonomic mobile robot is considered. Two novel control strategies are presented: a Lyapunov-based Control strategy, and a sliding mode control based on quasi-continuous sliding mode Controller for systems with relative degree greater than one. A complete stability analysis for both control strategies is presented. The proposed sliding mode controller matches perfectly with the system, and with a proper sliding surface choice, the line-following can be guaranteed including a specified line direction. Both strategies are experimentally validated, where the excellent transient performance can be verified.
\end{abstract}

Keywords: line following; unicycle; tracked robot; sliding mode; autonomous robotics

Palavras-chaves: seguimento de linha; uniciclo; robô com esteiras; modo deslizante; robótica autônoma

\section{INTRODUCTION}

The control of nonholonomic mobile robot, particularly unicyclelike robot is a topic very well studied by the control and robotic community. There exist several control strategies to deal with the trajectory tracking and path following control problem. The tracking problem is to design a control strategy that drives the mobile robot to a desired position at a predetermined time.

On the other hand, path following only requires the robot to converge to a reference curve (Do, 2015). Path following typicaly tends to achive smoother convergence and is less likely to push the control signals into saturation (Lapierre et al., 2006).

The problem for controlling unicycle-like ystems is that while moving on a plane, the system is not locally controllable (Lee et al., 2001), as a consequence the system cannot be controled by a smooth continuous state feedback control law in its original Cartesian representation (Brockett, 1983). In (Siegwart and Nourbakhsh, 2004) a strategy for point stabilization is to change the representation into polar coordinates. In (Casalino et al., 1995), besides point stabilization, path following is also considered in the same form. In (Lapierre et al., 2006) Lyapunov based and backstepping techniques are used to develop an adaptive control law to deal with vehicles uncertainties. In (Morro et al., 2011), the distance to the desired path is calculated using the projection of the robot position towards the desired path.

In the general, the proposed path following control strategies could presente very poor transient behavior, and an oscillation about the path could in general be observed. The oscillation behavior is mainly due to the nonholonomic constraints.

This paper presents two control strategies for line following problem, which can significally improve ths transient response. The first strategy considers a Lyapunov based design to develop a feedback control law. The second approach consists on a quasi-continuous sliding mode Controller.

\footnotetext{
1 This study was financed in part by $\mathrm{CNPq}$ and the Coordenacao de Aperfeicoamento de Pessoal de Nivel Superior - Brasil (CAPES) - Finance Code 88887.136349/2017-00 and 001.
}

\section{PROBLEM FORMULATION}

Consider a kinematic model of a unicycle robot described by:

$$
\left[\begin{array}{c}
\dot{x}_{r} \\
\dot{y}_{r} \\
\dot{\phi}_{r}
\end{array}\right]=\left[\begin{array}{cc}
\cos \left(\phi_{r}\right) & 0 \\
\sin \left(\phi_{r}\right) & 0 \\
0 & 1
\end{array}\right]\left[\begin{array}{c}
v \\
\omega
\end{array}\right],
$$

where $x_{r}$ and $y_{r}$ are the robot position on the plane, $\phi_{r}$ its orientation, $v$ is the linear velocity and $\omega$ angular velocity.

Here, the line following problem is considered in order to drive the unicycle to line with the same line orientation (see Fig. 1).

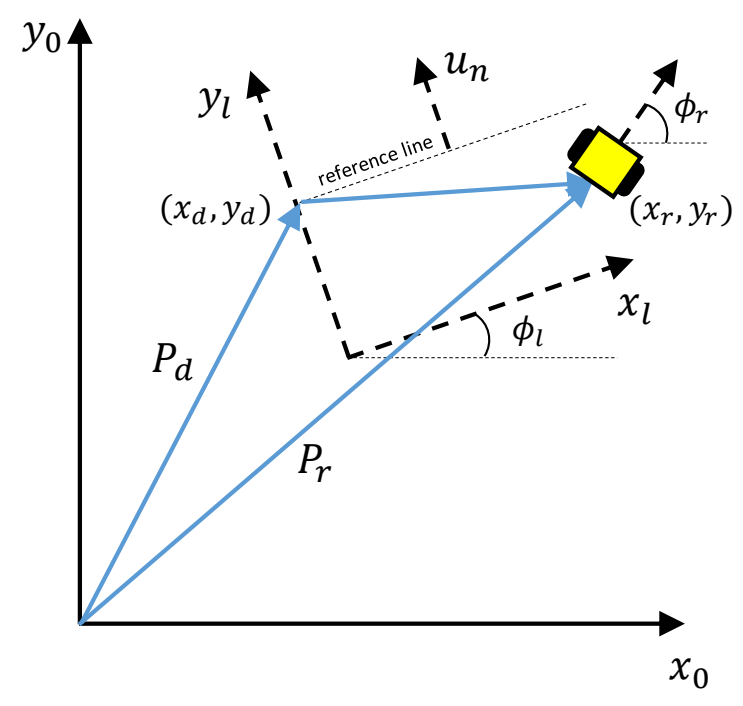

Figure 1. Geometric description of a unicycle robot

Consider that $1 P_{d}=\left[\begin{array}{ll}x_{d} & y_{d}\end{array}\right]^{T}$ is the position of any point $\left(x_{d}, y_{d}\right)$ belonging to the line, $P_{r}=\left[\begin{array}{ll}x_{r} & y_{r}\end{array}\right]^{T}$ is the robot position, $\phi_{l}$ is the line orientation, $\phi_{r}$ is the robot orientation and $u_{n}$ is a unit vector orthogonal to the line direction (all represented with respect to the inertial frame). 
The distance from the robot to the line can be defined as:

$$
l=u_{n}^{T}\left(P_{r}-P_{d}\right)
$$

and therefore,

$$
l=-\sin \left(\phi_{l}\right)\left(x_{r}-x_{d}\right)+\cos \left(\phi_{l}\right)\left(y_{r}-y_{d}\right)
$$

Considering that $P_{d}$ and $\phi_{l}$ are constants, the $l$ time-derivative can be written as:

$$
\dot{l}=-\sin \left(\phi_{l}\right) \dot{x_{r}}+\cos \left(\phi_{l}\right) \dot{y_{r}} .
$$

Then, substituting (1) in (3), one has

$$
i=-v \sin \left(\phi_{l}\right) \cos \left(\phi_{r}\right)+v \cos \left(\phi_{l}\right) \sin \left(\phi_{r}\right),
$$

and consequently

$$
i=v \sin \left(\phi_{r}-\phi_{l}\right) \text {. }
$$

Now, let $e_{\phi}=\phi_{r}-\phi_{l}$ be the orientation error with timederivative given by

$$
\dot{e}_{\phi}=\dot{\phi}_{r}-\dot{\phi}_{s}
$$

From (1), considering that $\dot{\phi}_{r}=\omega$, one has that

$$
\dot{e}_{\phi}=\omega \text {. }
$$

Finally the control system dynamics is given by

$$
\begin{aligned}
\dot{l} & =v \sin \left(e_{\phi}\right) . \\
\dot{e}_{\phi} & =\omega
\end{aligned}
$$

\section{CONTROL DESIGN}

In this paper is considered the problem of following the line for a given linear velocity $v$ while using the angular velocity $\omega$ as control input. The problem is to find an appropriate state feedback control law such that the closed loop system has stability properties guaranteed and convergence of the state variables $\left(l, e_{\phi}\right)$ to zero.

In this section two approaches are presented considering the system described in (4) under the following assumptions:

(A1) The linear velocity of the robot $v$ is a given positive constant;

(A2) The control input $u$ is the angular velocity of the robot $\omega$, i.e. $u=\omega$.

\subsection{Lyapunov Based Design}

The proposed feedback control law is given by the following equation:

$$
u=-k_{1} e_{\phi}-\frac{1}{e_{\phi}} k_{2} l v \sin \left(e_{\phi}\right)
$$

where $k_{1}$ and $k_{2}$ are positive arbitrary constants. It is important to notice that (5) is not singular at $e_{\phi}=0$. The stability analysis is presented in the following theorem.

Theorem 1. Consider the system (4) under the assumptions (A1)-(A2), and the control law (5). Then, the origin of closed loop system is asymptotically stable for $-\pi \leq e_{\phi} \leq \pi$.

Proof: Consider the following Lyapunov Function:

$$
2 V\left(l, e_{\phi}\right)=k_{2} l^{2}+e_{\phi}^{2} .
$$

The time derivative of $V$ is

$$
\dot{V}=k_{2} l i+e_{\phi} \dot{e}_{\phi}
$$

and therefore

$$
\dot{V}=k_{2} l v \sin \left(e_{\phi}\right)+e_{\phi} u \text {. }
$$

Note that using the control law (5), it follows that

$$
\dot{V}=-k_{1} e_{\phi}^{2} \leq 0 \text {. }
$$

The closed loop system is

$$
\left\{\begin{array}{l}
\dot{l}=v \sin \left(e_{\phi}\right) \\
\dot{e_{\phi}}=-k_{1} e_{\phi}-k_{2} l v \frac{\sin \left(e_{\phi}\right)}{e_{\phi}} .
\end{array} .\right.
$$

Since $V$ is continuously differentiable, radially unbounded, positive definite, and $\dot{V} \leq 0$ over the entire state space, by using LaSalle Invariance principle (Khalil, 1996), one has that all trajectories converge to the largest invariant set $\bar{\Omega}$ in $\Omega=$ $\left\{\left(l, e_{\phi}\right): \dot{V}=0\right\}=\left\{\left(l, e_{\phi}\right): e_{\phi}=0\right\}$. In the invariant set we have that $e_{\phi}=0$ and $i=0$. Besides that, to hold $e_{\phi}=0, \dot{e}_{\phi}=0$. Note that for the closed loop system (6), if both $\left(e_{\phi}, \dot{e}_{\phi}\right)=0$, thus $l=0$. Then, one can conclude that the only point within $\bar{\Omega}$ is the origin which is asymptoticly stable. Therefore, for any initial conditions $\left(l(0), e_{\phi}(0)\right)$, the system will eventually converge to $(0,0)$.

\subsection{Sliding Mode Control}

Noting that system (4) has relative degree equals two, since

$$
\ddot{l}=v \cos \left(e_{\phi}\right) u \text {. }
$$

Therefore, a normal approach for a sliding mode controller would not be ideal to the stated problem. Also, it is desired a chattering alleviated smooth control signal.

The solution adopted is to consider a Quasi-Continuous Sliding Mode Controller, firstly introduced by (Levant, 2005b). This controller has a continuous behavior until the sliding surface is reached, and once at the surface, a modulation function can be used to relieve the chattering.

The control law is given by

$$
u=-\alpha \frac{\dot{\sigma}+|\sigma|^{1 / 2} \operatorname{sign}(\sigma)}{|\dot{\sigma}|+|\sigma|^{1 / 2}} .
$$

The sliding variable is chosen as

$$
\sigma=l
$$

therefore its derivative is given by

$$
\dot{\sigma}=\dot{l}=v \sin \left(e_{\phi}\right) \text {. }
$$

The control law (7)-(9) is continuous everywhere except of the origin. It vanishes on the parabola $\dot{\sigma}+\beta|\sigma|^{1 / 2} \operatorname{sign}(\sigma)=0$. With sufficiently large $\alpha$ the closed loop trajectories enter the region between the curves $\dot{\sigma}+\beta|\sigma|^{1 / 2} \operatorname{sign}(\sigma)=0$ and cannot leave it (Levant, 2007).

Theorem 2. Consider the system (4) under the assumptions (A1)-(A2) and the quasi-continuous sliding mode control law (7) with sliding variable $\sigma$ and its derivative $\dot{\sigma}$ given by (8) and (9), respectively. Thus, closed loop system reaches the sliding surface $(\sigma, \dot{\sigma}) \equiv 0$ in finite time, and the origin is an attractive equilibrium point.

Proof: The proof is divided in two steps. Firstly, consider $e_{\phi} \in$ $[-\pi / 2, \pi / 2]$. In this scenario it is easy to show that the second derivative of the sliding variable

$$
\ddot{\sigma}=v\left|\cos \left(e_{\phi}\right)\right| \omega \text {. }
$$

Clearly, (10) can be represented in Filippov Diferential Inclusion (Levant, 2005a) as

$$
\ddot{\sigma} \in\left[K_{m}, K_{M}\right] \omega \quad \forall\left(l, e_{\phi}\right) \in\left(\mathbb{R}^{2}-\{(0,0)\}\right) .
$$


Therefore, by using the homogeneous high order sliding mode control law (7) and invoking Theorem 5 from (Levant, 2007) it is possible to conclude that with sufficiently large constant $\alpha$ the closed loop system is locally finite-time-stable.

On the second step, let the interval be $e_{\phi} \in(-\pi,-\pi / 2) \cup$ $(\pi / 2, \pi)$. In this case it is possible to conclude that the second derivative of the sliding variable becomes

$$
\ddot{\sigma}=-v\left|\cos \left(e_{\phi}\right)\right| \omega \text {. }
$$

Note that in this particular interval the control direction is inverted. Therefore, the neighborhood of equilibrium points where $e_{\phi}=(2 k+1) \pi$ for $k \in \mathbb{Z}$ are non attractive. To show this, consider the following transformation of the coordinate system:

$$
\left\{\begin{array}{l}
\bar{l}=l \\
\bar{e}_{\phi}=e_{\phi} \pm(2 k+1) \pi
\end{array}\right.
$$

Then, the dynamics of (13) is given by

$$
\left\{\begin{array}{l}
\dot{\bar{l}}=v \sin \left(\bar{e}_{\phi} \pm[2 k+1] \pi\right) \\
\dot{\bar{e}}_{\phi}=\omega
\end{array}\right.
$$

Let $V\left(\bar{l}, \bar{e}_{\phi}\right)$ be the following locally positive definite function

$$
2 V\left(\bar{l}, \bar{e}_{\phi}\right)=\bar{l}^{2}-\bar{e}_{\phi}^{2} .
$$

that satisfies $V\left(\bar{l}, \bar{e}_{\phi}\right)>0$ for all $\left(\bar{l}, \bar{e}_{\phi}\right) \subset \mathscr{D} \in \mathbb{R}^{2}$ such that $\mathscr{D}=\left\{\left(\bar{l}, \bar{e}_{\phi}\right):|\bar{l}|>\left|\bar{e}_{\phi}\right|\right\}$. Thus, for the closed loop system it can be found that

$$
\begin{aligned}
\dot{V}= & \bar{l} v \sin \left(\bar{e}_{\phi} \pm[2 k+1] \pi\right)+ \\
& +\bar{e}_{\phi} \alpha\left[\frac{v \sin \left(\bar{e}_{\phi} \pm[2 k+1] \pi\right)+|\bar{l}|^{1 / 2} \operatorname{sign}(\overline{1})}{\left|v \sin \left(\bar{e}_{\phi} \pm[2 k+1] \pi\right)\right|+|\bar{l}|^{1 / 2}}\right] .
\end{aligned}
$$

Note that, for instance, the test point $\left(\bar{l}, \bar{e}_{\phi}\right)=(1.5,0.5) \in \mathscr{D}$, $V\left(\bar{l}, \bar{e}_{\phi}\right)>0$ and $\dot{V}\left(\bar{l}, \bar{e}_{\phi}\right)>0$. Therefore, by invoking Chetaev Instability Theorem (Khalil, 1996) the origin of (14) in closed loop is unstable.

Thus, one can conclude that equilibrium points where $e_{\phi}=$ $(2 k+1) \pi \forall k \in \mathbb{Z}$ are unstable. In other words, in closed loop the system (4) has attractive equilibrium points at $\left(l, e_{\phi}\right)=$ $(0,2 k \pi) k \in \mathbb{Z}$.

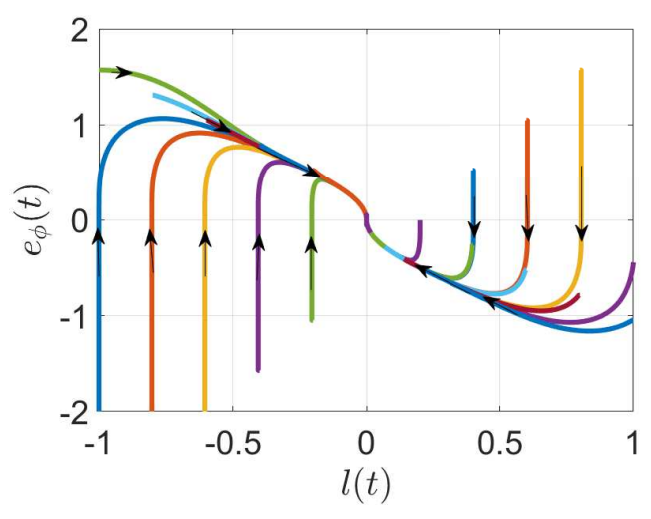

Figure 2. Phase portrait for the sliding mode controller.

From Theorem 2 one can ensure that for any initial conditions $\left(l(0), e_{\phi}(0)\right)$, the system will eventually converge to one of its attractive equilibrium points $(0,2 k \pi)$, that in practice are at the same. The trajectories are exemplified in Fig. 2.

\section{RESULTS}

Experimental results are obtained using a EOD (Explosive Ordnance) tracked robot (Fig. 3).

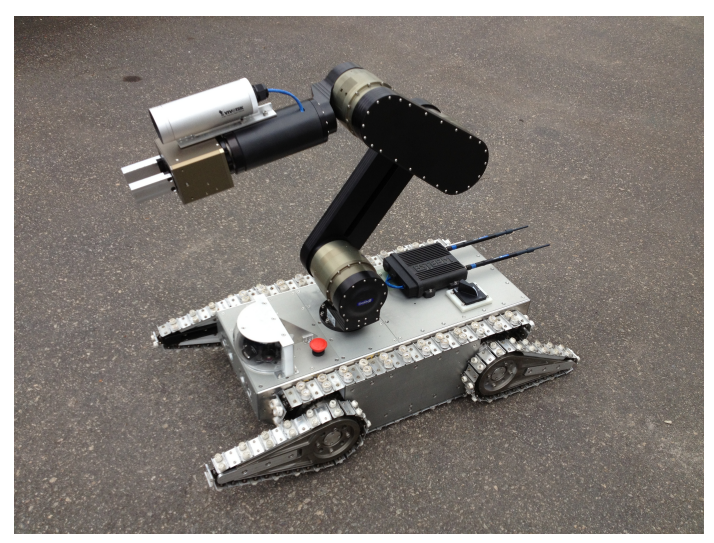

Figure 3. Tracked robot used in the experiments.

The robot traction is provided by two main tracks, actuated by independent Maxon EC45 motors. The motors are coupled to a Maxon GP52C gearbox with ratio 53:1 and are commanded by Maxon EPOS2 70/10 power drivers. The driver is capable to provide a very small rising time for the motors, making it a good match with the kinematic approach. The robot also carries an embedded $\mathrm{PC} / 104$ with an Intel Core $i 7$ processor, responsible for executing the control algorithm. All the software was developed using ROS framework. The robot position and orientation were both estimated with help of a Hokuyo UST-10LX laser sensor and Hector Slam rospackage.

Simulations are made using the kinematic model described in (1). Even knowing that the robot evidenced in Fig. 3 cannot be precisely described for this set of differential equations, the results have shown that the approximation is valid indeed.

In this section, experimental and simulated results are presented together on the same graphics. Two cases with different initial conditions are tested for both controllers. On the first experiment, in Figs. 4 and 6, easy initial conditions are considered. On the second, Fig. 5 and 7 we try to reproduce a problematic situation, trying to put the Theorem 2 in evidence.

On following results, the sliding mode controller presented in (7) is robust, given that besides bringing the sliding surface and its $(r-1)$ derivatives to zero, also guarantees convergence of the orientation error to even multiples of $\pi$. In practical therms, this fact means that for any set of initial conditions for the states, reference line is always followed in the correct direction of movement. This characteristic if greatly important to this work. Besides its robustness, in experiments, for systems with relative degree $r>1$ the condition where the sliding surface and its $(r-1)$ derivatives are zero is never reached, therefore making the control input practically continuous (Levant, 2005b).

The SMC parameters are $\alpha=3.75$ and $\beta=0.1$. In the Lyapunov based controller, $k_{1}=0.4$ and $k_{2}=20$. In all experiments, forward velocity of the robot is $v=0.1[\mathrm{~m} / \mathrm{s}]$ and the control signal is $u$. The control algorithm is executed with a frequency of $100 \mathrm{~Hz}$ and the initial conditions for the states $\left(l, e_{\phi}\right)$ are $(0.5,0)$ for the Figs. 4 and 6 and $(0.5,-\pi)$ for the Figs. 5 and 7. 
In Fig. 7, the trajectories are slightly away from each other, which could happen because the experimental $l$ remains constant at the beginning of the experiment. In this moment, the robot is basically rotating instead of moving forward.

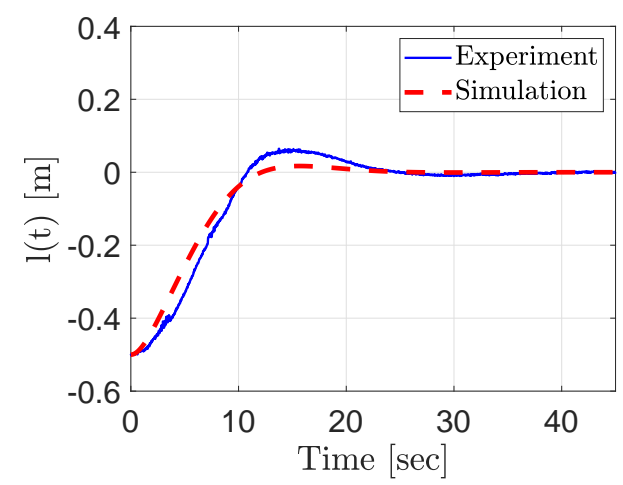

(a) Distance to the line (sliding surface).

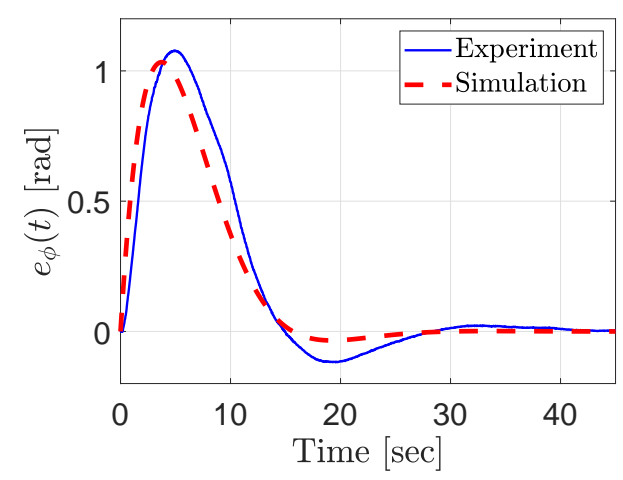

(b) Orientation error.

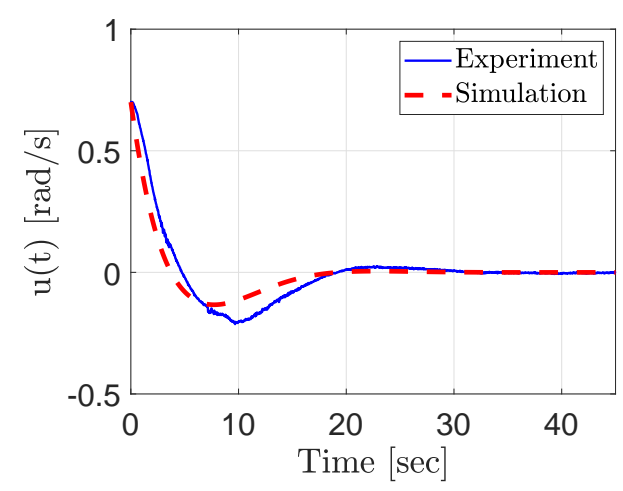

(c) Control input.

Figure 4. Results for Lyapunov based design controller with initial conditions $(0.5,0)$ and control law proposed in $(5)$.

\section{CONCLUSION}

In this paper the line-following problem for a nonholonomic mobile robot is considered. Two novel control strategies are presented: a Lyapunov-based Control strategy, and a sliding mode control based on quasi-continuous sliding mode Controller. A complete stability analysis for both control strategies is also presented. For both cases the states converge to zero with good transient behavior, and are robust to the robots unmodeled dynamics.

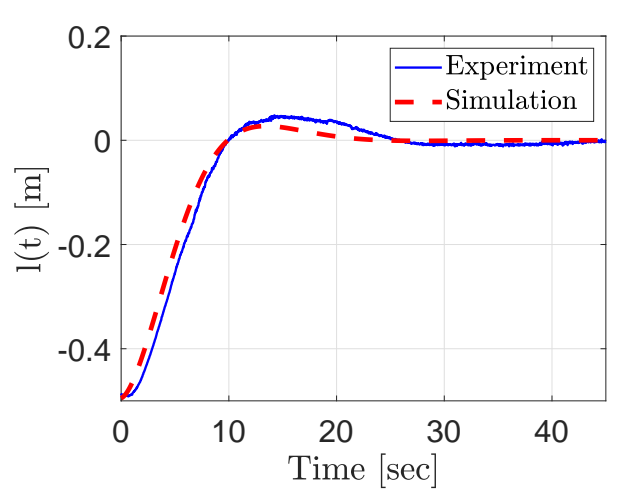

(a) Distance to the line (sliding surface).

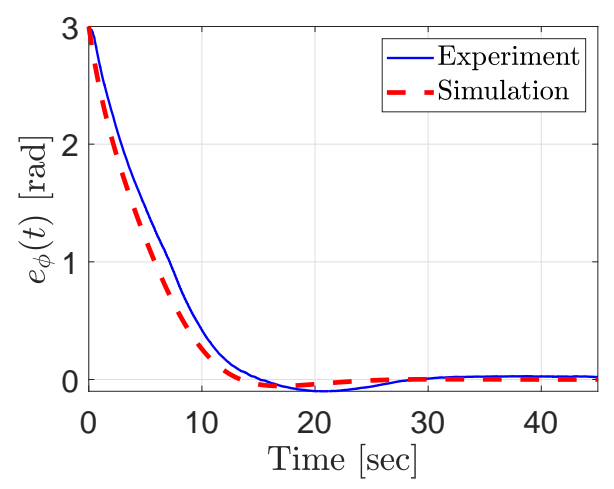

(b) Orientation error.

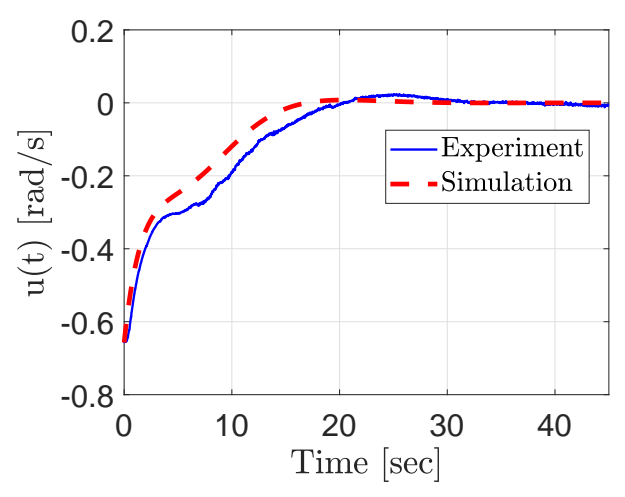

(c) Control input.

Figure 5. Results for Lyapunov based design controller with initial conditions $(0.5, \pi)$ and control law proposed in $(5)$.

As expected, the SMC is successful to drive the robot ti the line in the desired direction, even when the robot initial condition points towards an odd multiple of $\pi$ equilibrium point (This confirms that these equilibrium points are repulsive). A future work is to evaluate the use of this controller on a more general path following alternative.

\section{REFERENCES}

Brockett, R. (1983). Differential Geometric Control Theory. Boston: Birkhauser, Michigan.

Casalino, G., Aicardi, M., Bicchi, A., and Balestrino, A. (1995). Closed loop steering and path-following for unicycle-like vehicles: a simple lyapunov function based approach. IEEE Robotics and Automation Magazine, 2, 2735-2743. 


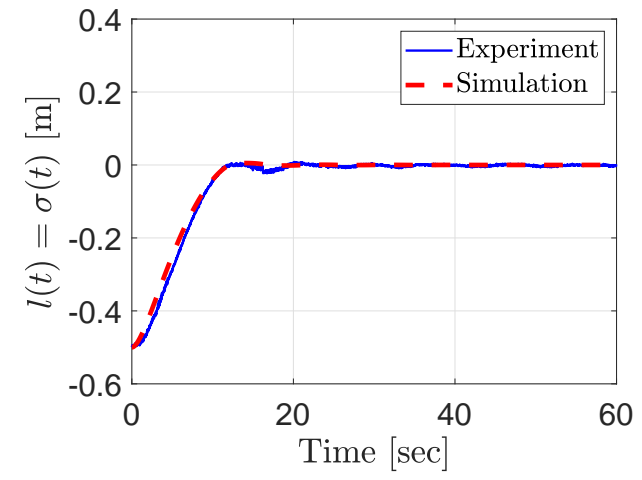

(a) Distance to the line (sliding surface).

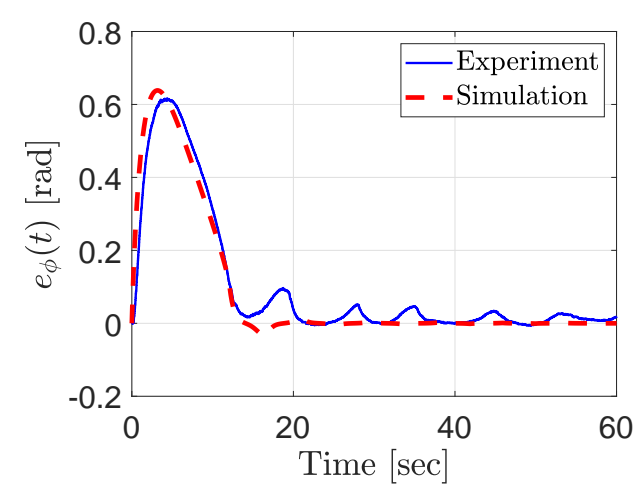

(b) Orientation error.

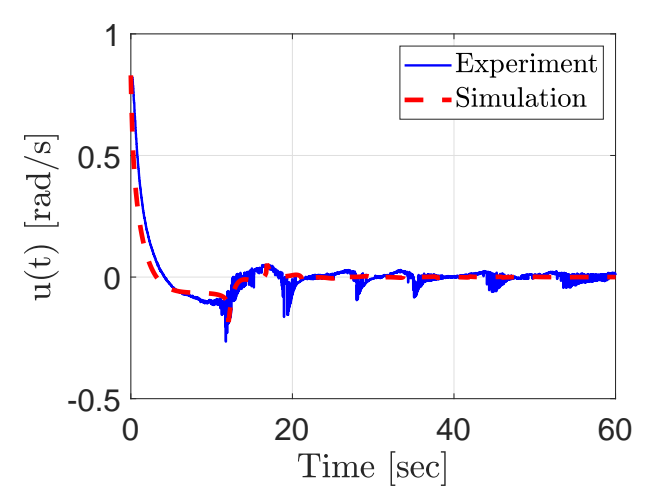

(c) Control input.

Figure 6. Results for sliding mode controller with initial conditions $(0.5,0)$ and control law proposed in $(7)$.

Do, K.D. (2015). Global output-feedback path-following control of unicycle-type mobile robots: A level curve approach. Robotics and Autonomous Systems, 74, 229-242.

Khalil, H.K. (1996). Nonlinear Systems. Prentice Hall, New Jersey.

Lapierre, L., Soetanto, D., and Pascoal, A. (2006). Nonsingular path following control of a unicycle in the presence of parametric modelling uncertainties. International Journal of Robust and Nonlinear Control, 16, 485-503.

Lee, T.C., Song, K.T., Lee, C.H., and Teng, C.C. (2001). Tracking control of unicycle-modeled mobile robots using a saturation feedback controller. IEEE Transactions on Control Systems Technology, 9, 305-318.

Levant, A. (2005a). Homogeneity approach to high-order sliding mode design. Automatica, 41, 823-830.

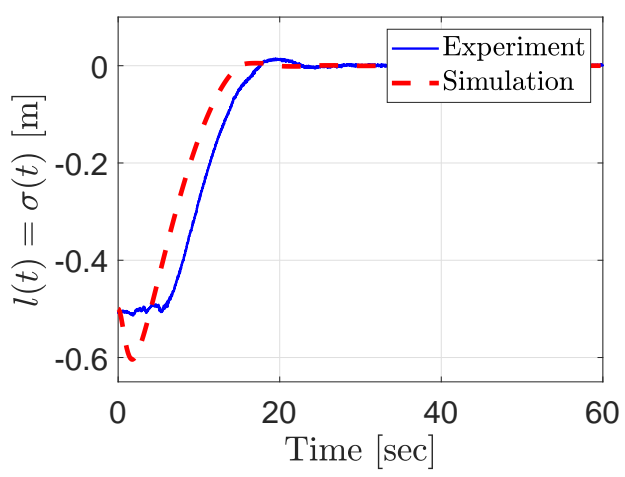

(a) Distance to the line (sliding surface).

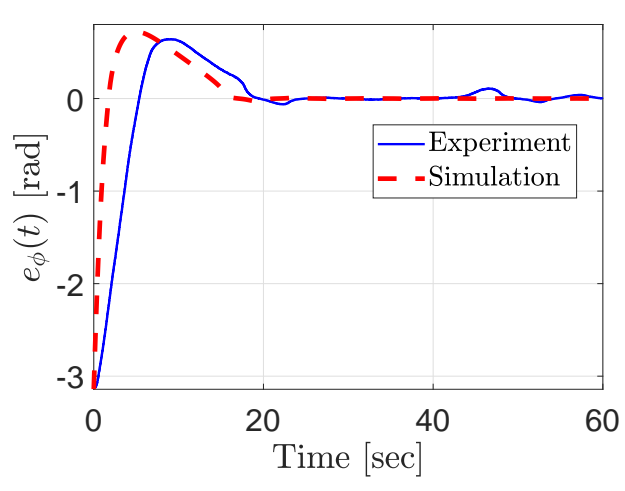

(b) Orientation error.

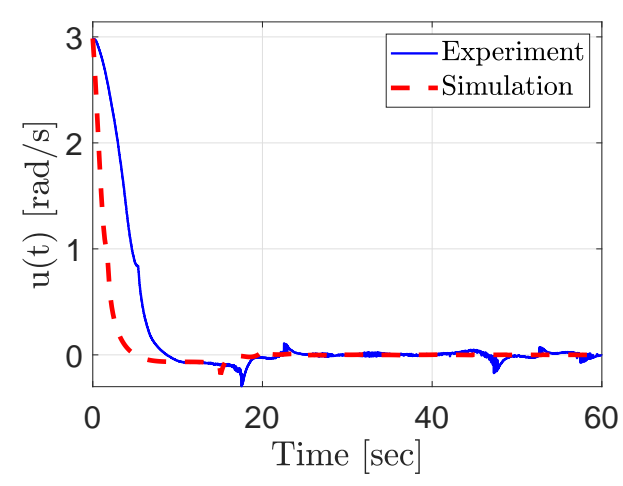

(c) Control input.

Figure 7. Results for sliding mode controller with initial conditions $(0.5, \pi)$ and control law proposed in (7).

Levant, A. (2005b). Quasi-continuous high-order sliding-mode controllers. IEEE Transactions on Automatic Control, 50, 1812-1816.

Levant, A. (2007). Principles of 2-sliding mode design. Automatica, 43, 576-586.

Morro, A., Sgorbissa, A., and Zaccaria, R. (2011). Path following for unicycle robots with an arbitrary path curvature. IEEE Transactions on Robotics, 27, 1016-1023.

Siegwart, R. and Nourbakhsh, I.R. (2004). Introduction to Autonomous Mobile Robots. Bradford Company Scituate, Massachusetts. 